\title{
Research on Intelligent City Problem Based on New Parameters
}

\author{
Peng Zhang*, Ke Zhang, Lei Dai, Yinhai Xu and Yunhao Dou \\ College of Mechanical and Electrical Engineering, Hohai University, No. 200 Jinling North Road, Changzhou City, \\ Jiangsu Province, China \\ ${ }^{*}$ Corresponding author
}

\begin{abstract}
We define an evaluation metric, known as Smart Growth Metric, to judge whether the development of cities from around the world meets smart growth, which means the higher Smart Growth Metric is, the deeper the extent of urban smart growth is. As regards of the selection of evaluation index, referred to factors widely used in literature and based on the ten principles of Smart growth theory and $3 E$ principles of sustainability, we select the most important 6 influence factors, respectively assigned to them, so as to arrive at a final metric. We set weight as secondary reference index with grey correlation so as to prove the correctness of target selection. Comprehensive evaluation is an extensive reference volume in the global background. Considering all factors with Entropy Method, we get new weight and new Smart Growth Metric by weight formula.
\end{abstract}

Keywords- smart growth metric; $3 E$ principles of sustainability; grey correlation

\section{INTRODUCTION}

In most countries, the city's development of economic, social infrastructure and other aspects has obvious advantages, which results in a rapid urbanization of the world. This will lead to the rapid increase of urban population, the scarcity of public infrastructure resources, as time passes, the world will be unable to give people a fair and sustainable home. So, how to solve the problem of urban sprawl and the loss of farmland around the city center will be the top priority of urban planning.

As a kind of urban planning theory, smart growth can effectively slow down the speed of urbanization of the world. The principles of smart growth: mix land uses, housing opportunities and choices, farmland, natural beauty and other development spaces' reservations and so on can be embodied as the factors which affect the success of the smart growth.

Our goal is to define a comprehensive evaluation metric, and combine with each city's population, demand, geographical conditions to develop a unique plan for smart growth. We base on the ten principles of smart growth and specifically consider six factors which including the density of population and non-urban land.

In the article, we define a metric of the smart growth index to measure the success of the city's smart growth. In our model, six factors are used to determine the final score by different standardized scores. According to the final score we can evaluate the success of the smart growth plan.
We present our general assumptions in Section 2, and some assumptions for a particular model are given in Section 4. In Section 3, we discuss our model and its validation. We also analyze the growth plans of the cities on two continents in detail and present our recommendations in this section. In addition, we discuss the potential and ranking of the growth plan in Section 3. Finally, we draw conclusions and discuss strengths and weaknesses of the growth plan in Section 5.

\section{METHODS}

\section{A. General Assumptions}

- The data we collect from the National Statistics Bureau is accurate and reliable. Because our data are all downloaded from the international organizations, it's reasonable to assume the high quality of their data.

- The data of the selected cities are random and objective, and there is no extreme data.

- Because the data coverage is narrow and the amount of data is small, on this basis, the error caused by the model is negligible.

\section{B. Total Scarcity Metric Model}

In this section, an evaluation metric is defined, we call it smart growth metric. It is quantified as a specific score, which can measure the success rate of smart growth of a city. A lower smart growth score means that the city's urbanization rate is too fast, exceeding its carrying capacity, does not meet the smart growth.

Firstly, we are short of the definition of smart growth. After considering the ten principles of the smart growth theory, we discover six influential factors that are related to it, which are as follows:

- Mix land use depends on the proportion of non-urban land use

- Creating a range of housing opportunities depends on new home sales

- The development of unique and attractive cities depends on the development of the local tourism industry

- Reservations to open spaces relate to forest cover

- The choice of transportation relate to the occupancy of private cars 
- The speed of urbanization depends on population density

Through the six factors we assign, we calculate and get our smart growth coefficient.

\section{Review of Literatures}

Literatures provide a wide range of factors, which affect the smart growth and the method of judging the success rate of smart growth. Therefore, before we build two models, we need to explain our ways to assign the six factors.

\section{Assessing Smart-Growth Strategies in Indian Cities: Grounded Theory Approach to Planning Practice}

This is realized by dividing the corresponding data by each factor to get the maximum possible score for the factor, (for example, if there are twenty factors in the data, 10 is the most appropriate score for the factor's most suiTABLE data) Then, we will normalize the score by multiplying the scores by 10 , so that the score of each factor can vary from 0 to 10 .

\section{1) SPSS Relevance Analysis}

First we will assign the normalized data. Through looking up relevant information, we get that each factor has the most appropriate standard. On this basis, we make the highest score 10 point, and the scores of the remaining data successively decrease until zero. The cumulative score of the six factors is the total score of the smart growth metric. Finally, associate the six scores with the total score, the results are shown in the following TABLE:

\section{TABLE I. CORRELATION ANALYSIS RESULTS OF SPSS}

\begin{tabular}{|l|l|l|l|l|l|l|}
\hline $\begin{array}{l}\text { Correlation } \\
\text { coefficient }\end{array}$ & $\begin{array}{l}\text { NUCLB } \\
\text { score }\end{array}$ & $\begin{array}{l}\text { PD } \\
\text { score }\end{array}$ & $\begin{array}{l}\text { NHT } \\
\text { score }\end{array}$ & $\begin{array}{l}\text { TI } \\
\text { score }\end{array}$ & $\begin{array}{l}\text { FCR } \\
\text { score }\end{array}$ & $\begin{array}{l}\text { NPC } \\
\text { score }\end{array}$ \\
\hline Total score & 0.580 & 0.606 & 0.064 & 0.586 & 0.461 & -0.038 \\
\hline $\begin{array}{l}\text { Whether it is } \\
\text { statistically } \\
\text { significant } \\
(<0.05)\end{array}$ & 0.051 & 0.001 & 0.052 & 0.752 & 0.049 & 0.849 \\
\hline
\end{tabular}

2) Gray System Correlation Analysis and Verification

We analyze the data of 20 groups with gray correlation, the correlation is the weight $\beta$ required.

While our Smart Growth metric $Y$ is as follows:

$$
\mathrm{Y}=\sum_{i=1}^{6} \beta_{i} * x_{i}
$$

The result is as follows:

TABLE II. RANK OF CORRELATION COEFFICIENT

\begin{tabular}{|l|l|c|c|c|c|c|}
\hline Coefficient & NUCLB & PD & NHT & TI & FCR & NPC \\
\hline Correlation & 0.7817 & 0.7851 & 0.7443 & 0.7242 & 0.7385 & 0.7763 \\
\hline Rank & 2 & 1 & 6 & 3 & 5 & 4 \\
\hline
\end{tabular}

The results is consistent with SPSS correlation analysis, so the accuracy of metric selection can be verified.

3) Model Validation

Therefore, we calculate the data of the remaining six groups of cities by the weight formula, compare the ranking of the resulting smart growth metric to the original, the results are consistent, therefore, the model is accurate.

TABLE III. TOTAL SCORE RANK

\begin{tabular}{|c|c|}
\hline Total Score & Rank \\
\hline 33.1046 & 3 \\
\hline 32.7945 & 4 \\
\hline 35.9873 & 1 \\
\hline 32.7524 & 5 \\
\hline 35.7026 & 2 \\
\hline 29.8324 & 6 \\
\hline
\end{tabular}

TABLE IV. WEIGHT METHOD RAN

\begin{tabular}{|c|c|}
\hline Total Score & Rank \\
\hline 0.8291 & 3 \\
\hline 0.6157 & 4 \\
\hline 1.1618 & 1 \\
\hline 0.3124 & 5 \\
\hline 1.0705 & 2 \\
\hline 0.2523 & 6 \\
\hline
\end{tabular}

\section{4) Conclusion}

Proof by weighting method, we can come to our definition of smart growth metric which can measure the success rate of urban smart growth.

TABLE V. EVALUATION CRITERIA

\begin{tabular}{c|c}
\hline Smart Growth Metric & The degree of success \\
\hline$<20$ & Very successful \\
\hline $20-40$ & More successful \\
\hline$>40$ & Not too successful \\
\hline
\end{tabular}

\section{Population Growth Supporting Measures Forecast}

\section{1) Establishment of MLRM}

Multivariate linear regression analysis model represents the dependent relationship between a phenomenon and a variety of other phenomena, a variety of other phenomena have an impact on the phenomenon. Let variable and variable $x_{1}, x_{2}, \cdots, x_{m}$ have a statistical relationship, $y$ is called the dependent variable, $x_{1}, x_{2}, \cdots, x_{m}$ is called the independent variable.

In this article, we use SGM as the dependent variable of the model, PD, NUCLB, FCR, NFM, II as the independent variable. Multiple Linear Regression Model is as follows:

$$
y=\beta_{0}+\beta_{1} x_{1}+\beta_{2} x_{2}+\cdots+\beta_{m} x_{m}+e
$$

Among the model, $\beta_{0}, \beta_{1}, \beta_{2}, \cdots \beta_{m}$ is an unknown parameter that is independent of the argument, is random error. 
Let the observations of section $n$ sample as $\left(y_{i}, x_{i 1}, x_{i 2}, \cdots x_{i m}\right) i=1,2, \cdots, n$

the formula we get after substituting the above formula is as follows:

$$
y_{i}=\beta_{0}+\beta_{1} x_{i 1}+\beta_{2} x_{i 2}+\cdots+\beta_{m} x_{i m}+e_{i}, i=1,2, \cdots n
$$

The regression parameters in the model can be estimated by the least squares method by using the sample data of the variables. The key of the parameter estimation is to obtain the correction value of the observed value, we define the value as, while there are wireless group of data meet the formula $y=A \beta+\ell$, where the sum of squares of only one set of the correction value is the smallest.

The parameters are estimated using the least squares method. The process is as follows:

Define the difference between the estimated value $\hat{y}$ and the original observation $y$ as $\hat{\ell}$

So, the error equation is as follows:

$$
\hat{\ell}=\hat{y}-y=A \hat{\beta}-y
$$

According to the least square estimation criterion, we can get that:

$$
\hat{e}^{T} \hat{e}=(\hat{y}-y)^{T}(\hat{y}-y)=\min
$$

In order to get the parameter estimates, constructor a formula, which is as follows:

$$
\varphi=\hat{e}^{T} \hat{e}=(A \hat{\beta}-y)^{T}(A \hat{\beta}-y)
$$

Calculate the partial derivative from to , let it be zero, you can meet the minimum conditions:

$$
\frac{\partial \varphi}{\partial \hat{\beta}}=(A \hat{\beta}-y)^{T} A=0
$$

According to the quality of column matrix derivation of column matrix, the least squares estimation of $\hat{\beta}$ is as follows:

$$
\hat{\beta}=\left(A^{T} A\right)^{-1} A^{T} y
$$

The least squares estimation of $y$ is as follows: $\hat{y}=A\left(A^{T} A\right)^{-1} A^{T} y$

\section{2) Model Calculation}

Through the standardization of the data model operation, the parameter estimation of $\hat{\beta}$ is shown in the following Table:

TABLE VI. TABLE VI: REGRESSION COEFFICIENTS

\begin{tabular}{|c|c|c|c|c|c|}
\hline Metric & PD & NUCLB & FCR & NFM & II \\
\hline$\hat{\beta}$ & 2.563 & -1.407 & -0.784 & 5.454 & 0.066 \\
\hline
\end{tabular}

According to the following diagram, we found that multiple linear regression line fitting results are better, the basic data are on both sides of the line.

\section{Normal P-P Plot of Regression Standardized} Residual

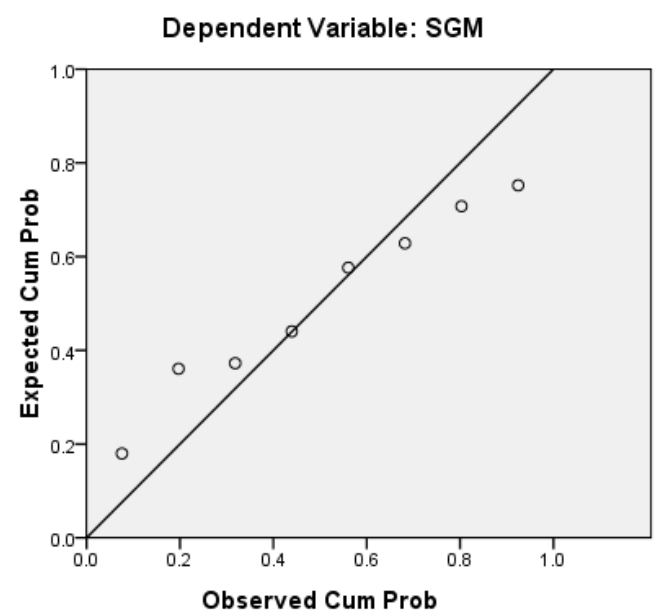

FIGURE I. LINEAR REGRESSION PLOT

\section{RESUlt AND DISCUSSION}

In this paper, we need to define a comprehensive evaluation metric, which can be widely used to measure the degree of smart growth in major cities around the world. And choose the Chinese city Karamay and the United States Oakland, combined with local geographic location, infrastructure investment and other specific facts to determine whether the smart growth plan to promote urban intelligence growth. After taking the particularity of population density, geographical location and economic development into account, we need to add some relative factors which have obvious differences, using the entropy method to determine the weights. In this way, we could develop a corresponding growth plan for Karamay and Oakland to deal with their urbanization. Firstly, basing on the article <Assessing Smart-Growth Strategies in Indian Cities: Grounded Theory Approach to Planning Practice $>$, we define the success rate of smart growth metric as SGM.

By considering the ten principles of smart growth and the sustainable there E principle, we identify six factors that affect 
the urban smart growth, and score each factor through the standardization principles, the final score would be the SGM reference score. When we get the SGM score, we will see it as a reference sequence, then the SPSS correlation analysis is used to analyze whether the six factors have the research and reference value, after that we use the gray correlation model to verify the correlation of six factors to the comprehensive metric. Our aim is to derive the weight $\beta_{i}$ of these six factors, and multiply them by the corresponding data $x_{i}$ to get the weight formulaY $=\sum_{i=1}^{6} \beta_{i} * x_{i}$, which will be the SGM's another reference score. Due to the limited lookup data, we choose a total of 26 worldwide cities, 20 sets of data are used as the reference of total score, the remaining 6 sets of data are used to verify the accuracy of the metric selection through the weight formula.

The model 1 we established is suitable for the general environment, while for the third and fourth question, we consider the population density, geographical location, infrastructure investment and other subtle factors of the two cities. We use the entropy weight method to analyze these factors, and get the weight which is more suitable for individual city. And our assessment for the level of urban smart growth, is more dependent on the score gotten from the weight formula. For the specific growth plan, we will formulate from the weight of model, and providing a most reasonable growth plan for the city as far as possible.

In order to cope with the population of each city will increase by an additional $50 \%$ by 2050 , we according to the data of major cities in recent years as a reference. And the multiple linear regression model is used to obtain the regression coefficients, in order to be the weight of forecasting the Smart Growth metric for 2050. As the urban population density is known, in order to stabilize the smart growth metric, we can get the predicted value of other influencing factors through the model.

Although our plan can theoretically help cities to successfully embark on the path of smart growth, however, under the background of obvious differences in economic development between countries, urbanization in some cities will continue to grow in the 30-40 years. We think that the theory of smart growth needs a long way to go, but it has a decisive role in restraining the growth of urban population, easing the excessive use of resources and rationalizing the infrastructure construction.

\section{REFERENCES}

[1] Li Xueming, Cheng Zhenjie, Cong Xueping. Analysis of physical geography background quality of urban development in Liaoning province [J]. Journal of Liaoning Normal University (Natural Science Edition), 2016, (1).

[2] Lin Guang. New Urbanism and American Urban Planning [J] American Studies, 2007, (4).

[3] Yang Xiu. Intelligent urbanization [J]. Journal of Urban Planning, 2015, (6).

[4] Peng Fangmei, Zhao Jiao. A Review of Theoretical Models and Empirical Research on Endogenous Urban Growth [J]. Economic latitude and longitude, 2010, (5).

[5] Huang Tianhang, Liu Ruilin, Dang Anrong. A Study on Intelligent Urban Development in Europe [J] .Residence, 2011, (6).
[6] http://web.b.ebscohost.com/ehost/detail/detail?vid=2\&sid=47ca1ee9-d 95a-41e9-9c22-9b9139216798\%40ssionmgr101\&hid=130\&bdata=Jmx hbmc9emgtY24mc2l0ZT1laG9zdC1saXZl\#AN=111078630\&db=a9h

[7] Ran Shuqing, Liu Xiaohui, Feng Yuwen. Study on the Spatial Differentiation of Economy, Population and Spatial Interaction in the Process of Metropolitan Development - A Case Study of Xi'an, Shaanxi Province [J]. Reform and Strategy, 2015, (2) 